\section{Attribute dimensions and patterns of trait inferences*}

\author{
MARK P. ZANNA, Princeton University, Princeton, N.J. 08540 \\ and \\ DAVID L. HAMILTON, Yale University, New Haven, Conn. 06510
}

Rosenberg, Nelson, \& Vivekananthan (1968) have suggested that Asch's (1946) "warm-cold" effect can be meaningfully interpreted within the framework of two dimensions (social and intellectual desirability) which underlie personality impressions. Their analysis suggested that the manipulation of unique information on one dimension would affect trait inferences on that dimension alone. This hypothesis was tested by replicating and extending Asch's experiment in terms of these two dimensions. The results strongly supported the hypothesis.

Recent research in social perception has been primarily concerned with investigating the processes by which people come to attribute personality traits to another person on the basis of minimal information. Following the early experiments by Asch (1946), one research problem has been to determine whether specification of the inferential relationships between personality traits would enable a priori predictions of which attributes will be inferred from any given set of stimulus traits.

In his most cited experiment, Asch (1946) characterized one stimulus person as "intelligent, skillful, industrious, warm, determined, practical, and cautious" (the "warm" condition) and another stimulus person as "intelligent, skillful, industrious, cold, determined, practical, and cautious" (the "cold" condition). Intuiting that the warm-cold varjable was a central dimension in impression formation, Asch predicted his manipulation would have a marked effect on trait inference. The results indicated that $S s$ in the "warm" condition did attribute different traits to the stimulus person than did Ss in the "cold" condition. However, this manipulation by no means affected all the antonym pairs presented.

Results of a study by Rosenberg, Nelson, \& Vivekananthan (1968) provide a basis for understanding why some, but not all, attributes were influenced by the warm-cold manipulation. These authors conducted a multidimensional scaling of personality trait words and identified two dimensions that appeared to underlie personality impressions. One dimension was named intellectual desirability and

*This study was supported in part by NSF Grant GS-3189 to the second author. reflected the extent to which the trait was viewed as desirable or undesirable in task-related activities. The second dimension was named social desirability and represented whether or not the trait was viewed as desirable in interpersonal activities. Results of the scaling showed that warm and cold essentially were polar adjectives on the social dimension, while the six context words used by Asch all represented the positive pole of the intellectual dimension. Hence, warm and cold conveyed unique information about the stimulus person on the social dimension. Rosenberg et al's analysis also indicated that the effect of varying warm vs cold appeared to be limited to those response traits which also fell on the social desirability dimension.

This interpretation suggests that differential patterns of trait inferences will occur when the manipulation of a stimulus attribute varies unique information about the persons to be judged. Furthermore, it suggests that such differential inferences will be made only for those attributes falling on the same dimension as the manipulated trait variable. The present experiment tested this interpretation by replicating and extending Asch's original study. Two pairs of stimulus persons, based on the dimensions reported by Rosenberg et al (1968), were created. Within each pair, information on one dimension was manipulated in a context of attributes from the positive pole of the other dimension. The basic hypothesis was that, for each pair of stimulus persons, the manipulation of unique information on one dimension would affect trait inferences on that dimension alone.

\section{SUBJECTS} college-age males who signed up to participate in psychological research. Ss were run in groups ranging in size
The Ss in the experiment were 70 from 4 to 13 and were paid for their participation. Within each experimental session, Ss were assigned randomly to one of the four conditions.

\section{MATERIALS}

Two pairs of stimulus persons were created, each stimulus person being described by five attributes. In one pair (the "warm" and "cold" conditions), the social-dimension attributes warm and cold were presented in the context of four positive intellectual traits: industrious, skillful, determined, and practical. In the other pair (the "industrious" and "lazy" conditions), polar attributes on the intellectual dimension, industrious and lazy, were included in a positive social context consisting of warm, sociable, good-natured, and humorous.

The stimulus persons were rated on 20 trait inference scales. The response traits included five traits from each pole of Rosenberg et al's (1968) two dimensions. The sequence of these response traits was such that no two consecutive words were from the same dimension.

\section{PROCEDURE}

When Ss arrived for the experiment, they were given one of four experimental booklets. The first page, with the title "Study of Impression Formation" at the top, gave the following instructions: "This study is concerned with the way people form a first impression of another person... You will be given a series of terms describing the personality attributes of a young man who, for convenience, we'll call Bob. From this information we would like you to form a first impression of Bob ...."

The next page presented the five stimulus traits which characterized Bob. On this page, Ss were asked to "consider these traits carefully, think about the kind of person Bob is, and then write a description of your first impression of Bob ..." This procedure was included as an attempt to guarantee that Ss did, in fact, form impressions of the target persons.

After Ss completed their descriptions, they were presented the response traits with the following instructions: "On the next page there are several additional attributes which might or might not be used to characterize Bob's personality. For each of these attributes, indicate ... how probable it is that Bob is also described by that trait."

Each response trait was followed by an 11-point probability scale that ranged from .00 to 1.00 in .10 intervals. The endpoints, .00 and 1.00 , were labeled "impossible" and "certain," respectively. The midpoint, .50 , was labeled "uncertain." 1

When Ss completed this final task, 


\begin{tabular}{|c|c|c|c|c|c|c|}
\hline \multirow[b]{2}{*}{ Trait Category } & \multicolumn{5}{|c|}{ Condition } & \multirow[b]{2}{*}{ t } \\
\hline & $\begin{array}{c}\text { Warm } \\
(N=18)\end{array}$ & $\begin{array}{c}\text { Cold } \\
(N=17)\end{array}$ & $\mathbf{t}$ & $\begin{array}{l}\text { Industrious } \\
\qquad(\mathrm{N}=18)\end{array}$ & $\begin{array}{c}\text { Lazy } \\
(N=17)\end{array}$ & \\
\hline Intellectually Desirable & $.69^{*}$ & .69 & $<1$ & .61 & .44 & $4.01 *$ \\
\hline Intellectually Undesirable & .16 & .20 & $<1$ & .30 & .41 & $2.29 \dagger$ \\
\hline Socially Desirable & .68 & .46 & $5.79 * *$ & .75 & .75 & $<1$ \\
\hline Socially Undesirable & .33 & .56 & $4.48 * *$ & .24 & .25 & $<1$ \\
\hline
\end{tabular}

* Mean probability that the target person possesses traits in a given category.

$* * p<.001 ;+p<.05$

the $E$ explained the purpose of the study, the rationale underlying the procedures, and answered any questions the Ss had. RESULTS

The results of the "warm" and "cold" conditions are presented in the left half of Table 1 . Here it can be seen that unique social-dimension information conveyed by the terms warm and cold did affect inferences Ss made about what additional traits Bob was likely to possess. However, differential inferences occurred only for those response traits taken from the social desirability dimension. Overall, Ss in the "warm" condition felt that Bob was more likely to have socially desirable attributes $(\mathrm{p}<.001)^{2}$ and less likely to have socially undesirable traits $(p<.001)$ than did Ss in the "cold" condition. Analyses of individual traits revealed that Ss in the "warm" condition judged Bob to be more sincere ( $p<.01)$, helpful $(p<.01)$, tolerant $(\mathrm{p}<.12)$, popular $(\mathrm{p}<.001)$, and happy $(\mathrm{p}<.001)$, as well as less pessimistic $(\mathrm{p}<.10)$, unpopular $(p<.01)$, unhappy $(p<.001)$, irritable $(p<.01)$, and moody $(p<.10)$ than did Ss in the "cold" condition.

In contrast, traits on the intellectual desirability dimension were not affected by the warm-cold manipulation (overall, $t<1$, for both the positive and negative traits). In both conditions, Ss strongly characterized Bob with intellectually desirable traits.

A second test of the hypothesis is provided by the results of the "industrious" and "lazy" conditions which are presented in the right half of Table 1. Unique information on the intellectual desirability dimension influenced the pattern of trait inferences, but again only for traits that fell along the predicted dimension. Overall, $S s$ in the "industrious" condition felt that Bob was more likely to possess intellectually desirable traits $(p<.001)$ and less likely to have intellectually undesirable traits $(p<.05)$ than Ss in the "lazy" condition. Analyses of individual traits revealed significant differences for 5 of the 10 traits along this dimension; of the other 5 traits, 4 were ordered in the prediction direction. Ss in the "industrious" condition reported that Bob was more likely to be scientific ( $\mathrm{p}<.01)$, discriminating $(\mathrm{p}<.01)$, and persistent $(p<.001)$, as well as less likely to be foolish $(\mathrm{p}<.01)$ and irresponsible $(p<.01)$ than $S s$ in the "lazy" condition.

In contrast, traits on the social desirability dimension were not affected by the manipulation (overall, $t<1$, for both the positive and negative traits). In both conditions, Ss strongly characterized Bob with socially desirable traits.

\section{DISCUSSION}

The results of the present experiment provide strong support for the basic hypothesis. Unique information on either the social or intellectual desirability dimension strongly influenced the nature of the traits that were attributed to the stimulus person. The findings, therefore, indicate the importance of considering different attribute dimensions in understanding patterns of trait inference.

In interpreting his results, Asch (1946) argued that the warm-cold variable was a "central trait." The present findings also support Rosenberg et al's (1968) analysis of the meaning of this concept. Centrality is not a property of certain traits, but-rather-is a function of the inferential relationships among a set of traits. In the present experiment, warm and cold provided unique social-dimension information. Consequently, inferences on social-dimension response scales were heavily influenced. The present results also demonstrate that this effect is specific to the social dimension (i.e., inferences on intellectual-dimension scales were not influenced by the warm-cold manipulation). They also show that comparable effects can be observed on the intellectual dimension by manipulation of information unique to that dimension.

These results also have implications for research on impression formation models. These models attempt to account for ratings of stimulus persons in terms of properties of the individual stimulus elements describing the persons. To date, the fact that stimulus traits can represent different dimensions of information has not been recognized in experimental designs. The judgment scale used in these studies (typically, the likableness of a stimulus person) may reflect one dimension of information (e.g., social) more than another. If so, then the present findings suggest that attributes representing that dimension of content should have more influence (i.e., receive greater weight) than other attributes in the judgment process.

\section{REFERENCES}

ASCH, S. E. Forming impressions of personality. Journal of Abnormal \& Social Psychology, 1946, 41, 258-290.

ROSENBERG, S., NELSON, C., \& VIVEKA NAN THAN, P. S. A multidimensional approach to the structure of personality impressions. Journal of Personality \& Social Psychology, 1968, 9, 283-294. NOTES

1. It should be noted that $S$ s were not asked to choose between antonym pairs as in past research. Instead, response traits were presented separately to increase the precision of the dependent measures.

2. All probability levels reported are based on two-tailed tests. 